\title{
Centrifuge testing to evaluate the liquefaction response of air-injected partially saturated soils beneath shallow foundations
}

\author{
A. $\operatorname{Zeybek}^{1}$ (D) S. P. G. Madabhushi
}

Received: 22 January 2016/Accepted: 7 July 2016/Published online: 21 July 2016

(C) The Author(s) 2016. This article is published with open access at Springerlink.com

\begin{abstract}
Earthquake-induced liquefaction of saturated soils continues to cause severe damage to structures with shallow foundations. In recent years, artificially reducing the degree of saturation and forming partially saturated zones within saturated soils has been proposed as a liquefaction mitigation technique. This study experimentally investigates the liquefaction response of air-injected partially saturated soils beneath shallow foundations. A series of centrifuge tests were conducted on the shallow foundations with different bearing pressures. The results of the tests show that the generation of excess pore pressures and consequent liquefaction-induced settlements of shallow foundations were a strong function of the degree of saturation. Forming spatially distributed partially saturated zones in the liquefiable soils limited the development of high excess pore pressures and liquefaction susceptibility of soils, particularly at the higher confining stresses. The reduction in the degree of saturation of soils decreased the depth of liquefied soil layer, and increased the resistance of soil to the bearing capacity failure. On the other hand, the decrease in the degree of saturation of liquefiable soils led the larger accelerations to be transmitted to the foundations through unliquefied soil zones. It is therefore concluded that use of air-injection as a liquefaction mitigation measure does reduce structural settlements, but will have the consequence of larger structural accelerations.
\end{abstract}

Keywords Geotechnical centrifuge - Liquefaction and mitigation · Air injection - Shallow foundations · Partially saturated soils · Degree of saturation

A. Zeybek

az323@cam.ac.uk

S. P. G. Madabhushi

mspg1@cam.ac.uk

1 Schofield Centre, University of Cambridge, Cambridge CB3 OEL, UK

2 Department of Engineering, University of Cambridge, Cambridge CB2 1PZ, UK 


\section{Introduction}

The liquefaction or softening of the foundation soils have repeatedly caused severe damages to structures with shallow foundations during earthquakes, such as recently during the 2010 Maule, Chile and the 2011 Christchurch, New Zealand earthquakes. The loose, saturated soils may undergo liquefaction during earthquake loading, depending on several parameters such as the relative density $\left(\mathrm{D}_{\mathrm{r}}\right)$, degree of saturation $\left(\mathrm{S}_{\mathrm{r}}\right)$, effective confining stress $\left(p^{\prime}\right)$ and the boundary conditions that determine the pore fluid drainage. The liquefaction of soils results in a decrease in the effective stress and the associated shear strength and stiffness degradation. When the excess pore pressures are built up to cause full liquefaction (near-zero effective stress state) beneath the shallow foundations, significant deformations including excessive settlement, tilting and shifting of overlying structures can occur. In recent years, some researchers have been investigating liquefaction mitigation techniques that involve the artificial introduction of gas bubbles and creating partially saturated zones in the liquefiable soil deposits. Some of these techniques are water electrolysis (Yegian et al. 2007), drainage-recharge (Takemura et al. 2008) and air injection (Okamura et al. 2011).

Natural soils below the ground water table are usually considered to be fully or nearly fully saturated (Tsukamoto et al. 2002). However, partially saturated soils can form as a consequence of biological activities in the nature (Mitchell and Santamarina 2005), or they can be artificially formed by the introduction of gas bubbles. The degree of saturation of such soils is obviously lower, and the reduction in the degree of saturation is attributed to the existence of retained air bubbles. It must be pointed that there is a difference between unsaturated soils with a vadose zone above the water table due to the capillarity and partially saturated soils below the water table with the presence of trapped air bubbles. In this paper, the focus will be on the latter. The influence of degree of saturation on the liquefaction resistance of soils has been investigated by a number of researchers through laboratory tests, and the associated test results have shown that the liquefaction resistance of saturated soils is markedly increased by even a small decrease in the degree of saturation (e.g. Yoshimi et al. 1989; Tsukamoto et al. 2002).

In addition to the laboratory tests, some physical model tests have been conducted to study the liquefaction behaviour of air-injected partially saturated soils. The research particularly investigating the response of shallow foundations resting on these soils has suggested that in accordance with the laboratory test data, the liquefaction potential of liquefiable soils and relevant structural settlements significantly decrease as the degree of saturation reduces (e.g. Marasini and Okamura 2015). Most of this research was qualitative in nature. Zeybek and Madabhushi (2016) have investigated in a quantitative fashion the deformation mechanisms under shallow foundation by creating partially saturated zone in the soil beneath using air injection. This research showed the efficacy of air-injection in reducing the settlements and focused on identifying the changes in the deformation mechanisms going from nearly fully saturated to partially saturated case.

In the experimental research represented here, the degree of saturation following air injection was determined based on the volume of air bubbles retained in the soil pores. It will be shown that the soil deformations reduce both in magnitude and the depth to which they occur below the ground surface, with a decrease in the degree of saturation. The experimentally observed deformations using the Geo-PIV (Particle Image Velocimetry) software (White et al. 2003) confirm these observations. In addition, it will be shown that as a direct consequence of reduction in the degree of saturation, the accelerations recorded 
by the shallow foundation increase. These results were confirmed for two sets of foundations with different bearing pressures, one classed as 'light' and other as 'heavy' (see Table 1).

\section{Experimental methodology}

A series of six centrifuge experiments were performed. The Turner beam centrifuge at Schofield Centre in Cambridge University was used in the experiments. The detailed descriptions of the Schofield Centre geotechnical centrifuge facilities as well as instrumentation were given by Madabhushi (2014). Centrifuge models were prepared and spun at a nominal centrifugal acceleration of $70 \mathrm{~g}$. All measurements presented in this paper are at prototype scale, unless otherwise indicated. Two bench-mark centrifuge tests, namely FSH1 and FSL-1, were carried out on the nearly fully saturated soils $\left(S_{\mathrm{r}}=99.0 \%\right)$ beneath the heavy and light shallow foundations with bearing pressures of 135 and $50 \mathrm{kPa}$, respectively. Other four tests (PSH-1, PSH-2 and PSL-1, PSL-2) were performed on the air-injected partially saturated soils with varying degrees of saturation beneath the same heavy and light foundations. Table 1 provides a summary of the centrifuge testing programme.

The soil used in the experiments was Hostun $\mathrm{HN} 31$ sand $\left(\mathrm{d}_{10}=0.315 \mathrm{~mm}\right.$, $\mathrm{d}_{50}=0.480 \mathrm{~mm}, \mathrm{U}_{\mathrm{c}}=1.67, \mathrm{e}_{\min }=0.555, \mathrm{e}_{\max }=1.01$ and $\mathrm{G}_{\mathrm{s}}=2.65$ ). Hostun HN31 sand was dry pluviated in a thick Perspex window box to attain $240 \mathrm{~mm}$ deep homogenous, liquefiable soil beds at a relative density of $40 \%$. The total soil depth simulates $16.8 \mathrm{~m}$ liquefiable material in prototype scale. A rubber air curtain hose, with several tiny openings of about $0.5 \mathrm{~mm}$ diameter and $5 \mathrm{~mm}$ spacing, was used at the bottom of sand beds to inject air bubbles into the saturated soil. Arrays of piezo-electric accelerometers, micro-electromechanical system (MEMS) accelerometers and pore pressure transducers were positioned at the desired locations to measure the soil/foundation accelerations and pore pressure response in the soil. Linear variable differential transducers (LVDT) were also used to measure the foundation and free-field settlements at different locations. The schematic illustration of centrifuge models was shown in Fig. 1.

The dry sand models were then saturated using CAM-sat system, as described by Stringer and Madabhushi (2009). During the saturation process, aqueous solutions of

Table 1 Centrifuge testing programme

\begin{tabular}{|c|c|c|c|c|c|c|}
\hline \multirow{2}{*}{$\begin{array}{l}\text { Test } \\
\text { ID }\end{array}$} & \multirow[t]{2}{*}{ Model identification } & \multirow{2}{*}{$\begin{array}{l}\text { Input motion } \\
\text { features }\end{array}$} & \multicolumn{4}{|c|}{ Test conditions } \\
\hline & & & $\begin{array}{l}\text { Initial } \\
\text { relative } \\
\text { density } \\
\%)\end{array}$ & $\begin{array}{l}\text { Initial } \\
\text { degree of } \\
\text { saturation } \\
(\%)\end{array}$ & $\begin{array}{l}\text { Final degree of } \\
\text { saturation after } \\
\text { air injection } \\
(\%)\end{array}$ & $\begin{array}{l}\text { Foundation } \\
\text { bearing } \\
\text { pressure } \\
(\mathrm{kPa})\end{array}$ \\
\hline FSH-1 & $\begin{array}{l}\text { Nearly fully } \\
\text { saturated }\end{array}$ & $\begin{array}{l}\text { Peak input } \\
\text { acceleration }\end{array}$ & & & 99.0 & 135 \\
\hline PSH-1 & Partially saturated & $0.18 \mathrm{~g}$ & & & 89.0 & 135 heavy \\
\hline PSH-2 & Partially saturated & & 40.0 & 99.0 & 86.0 & 135 \\
\hline FSL-1 & $\begin{array}{l}\text { Nearly fully } \\
\text { saturated }\end{array}$ & Frequency & & & 99.0 & 50 \\
\hline PSL-1 & Partially saturated & $0.72 \mathrm{~Hz}$ & & & 93.1 & 50 light \\
\hline PSL-2 & Partially saturated & & & & 79.5 & 50 \\
\hline
\end{tabular}




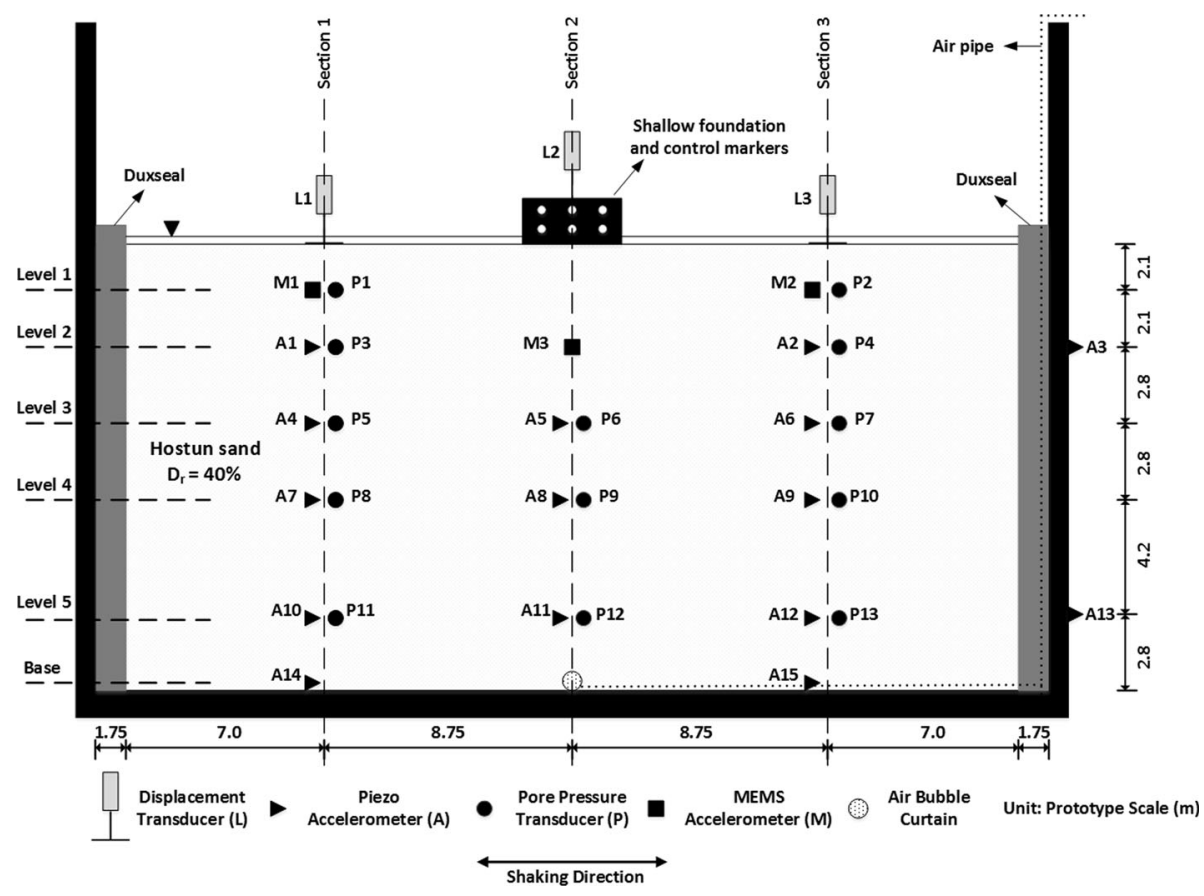

Fig. 1 Centrifuge model layout in experiments

hydroxypropyl methylcellulose (HPMC) with a viscosity of 70 times that of water were first prepared as the pore fluid to avoid any incompatibility between dynamic and diffusion time scaling laws (Schofield 1981). The models were enclosed and placed under vacuum at approximately $-90 \mathrm{kPa}$. They were then flushed with $\mathrm{CO}_{2}$ three times. Using the CAMSat system, the models were saturated very slowly with the prepared viscous pore fluid. The degree of saturation of models was determined based on the conventional mass and volume method. The computed degrees of saturation at the end of the saturation process were consistently above $99 \%$. It is of significance to note that although the masses, volumes and densities of the pore fluid and solids were meticulously determined, certain errors in the computed saturation ratios were still expected to occur due to the sensitivity of the conventional method (Kutter 2013). The phreatic surface of the saturated models was kept at approximately $0.35 \mathrm{~m}$ above the ground surface after spinning. Following the saturation, the saturated models were placed on the Turner beam centrifuge carefully. Shallow foundation models with the same dimensions ( $3.5 \mathrm{~m}$ width and $1.75 \mathrm{~m}$ height) and instrumented with MEMS accelerometers were carefully placed on the ground surface. The foundations representing a shallow foundation of a heavy structure were made of brass and exerting a bearing pressure of $135 \mathrm{kPa}$ at $65 \mathrm{~g}$, whereas those representing a shallow foundation of a light structure were made of duralumin and applying a bearing pressure of $50 \mathrm{kPa}$ at $65 \mathrm{~g}$. After all, the centrifuge was started, and the centrifugal acceleration was increased in steps of $10 \mathrm{~g}$ to the targeted $\mathrm{g}$ level $(65 \mathrm{~g}$ at the foundation level and $70 \mathrm{~g}$ at the base of the model). In the saturated soil tests with $\mathrm{S}_{\mathrm{r}}=99.0 \%$ (FSH-1 and FSL-1), the earthquakes were straightaway applied. However, in the partially saturated soil tests (PSH1 and -2 and PSL-1 and -2), air was first injected at a pressure of approximately $180 \mathrm{kPa}$ at the base of the model to reduce the degree of saturation, and then the models were shaken 
after $5 \mathrm{~min}$ from the end of the air injection process. The earthquakes were applied using a stored angular momentum (SAM) actuator device (Madabhushi et al. 1998), and they were parallel to the long side of models. Input acceleration-time histories recorded during the tests are presented in Fig. 2. The amplitude and frequency of earthquakes were same, and the peak base acceleration of around $0.18 \mathrm{~g}$ was used for all tests. However, the duration of shakings was much longer than usual (28s) in the tests PSL-1 and PSL-2 to investigate the behaviour of air-injected partially saturated soils during the prolonged shaking. In the presentation of the test results, only the input acceleration data from the tests FSH-1 and FSL-1 are illustrated for the heavy and light structure models, respectively. This permits making a direct comparison between the tests and comparing general patterns of behaviour. During the tests, digital images were acquired using a high speed camera to investigate the soil deformations beneath the shallow foundations through particle image velocimetry (PIV) technique. The preparation of air-injected partially saturated soils and image analysis were discussed in detail elsewhere (Zeybek and Madabhushi 2016).

\section{Seismic response}

Observations of soil response beneath the shallow foundations and in the free-field shed light on the influence of degree of saturation on the settlement and rate of settlement, excess pore pressure generation and dissipation as well as soil deformation mechanisms. The quantitative evaluation of these parameters might be a necessary step toward the effective application of liquefaction mitigation techniques using air-injection.

\subsection{Response of soil beneath shallow foundations}

\subsubsection{Settlement}

The average foundation settlement-time histories measured in all experiments, together with the input acceleration-time histories recorded during the tests FSH-1 and FSL-1 are
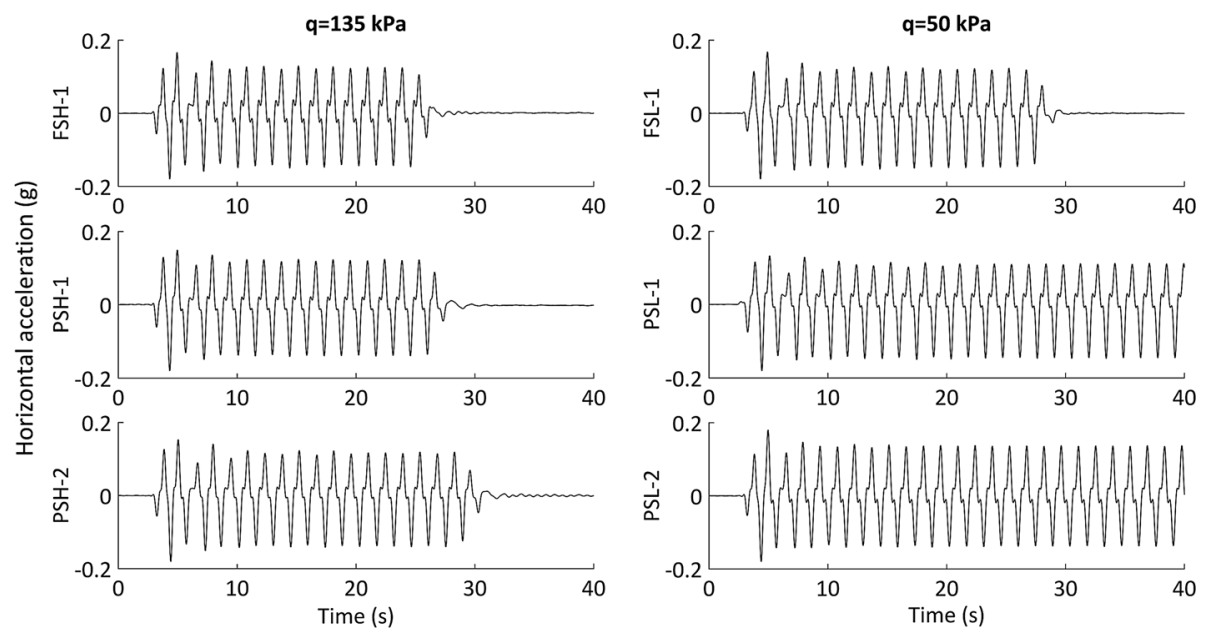

Fig. 2 Input horizontal acceleration-time histories recorded in all tests 

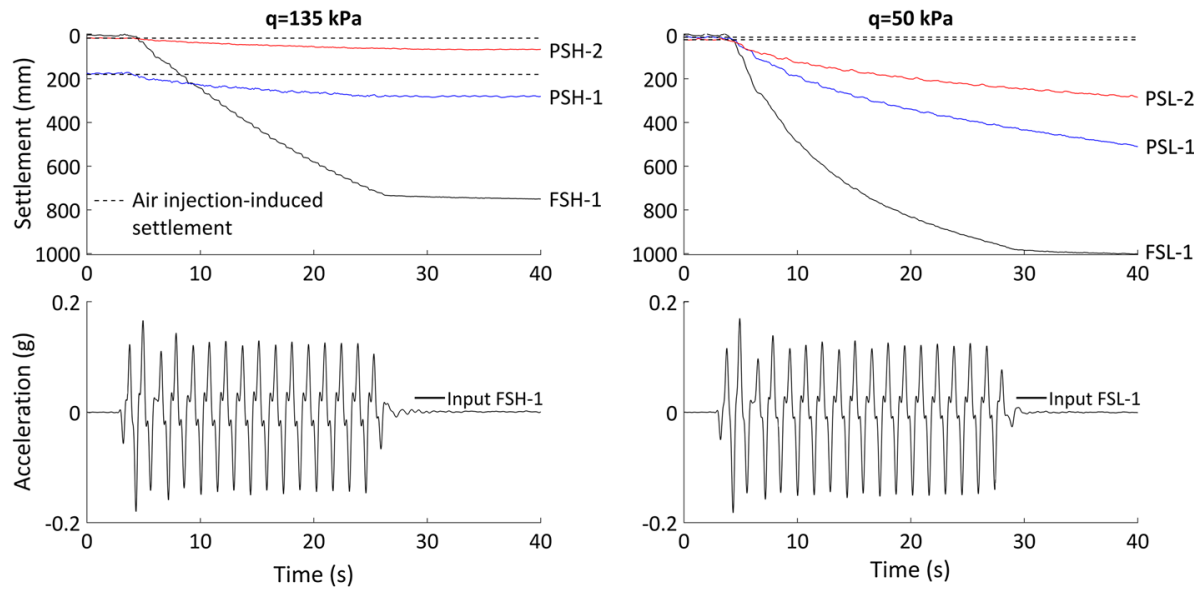

Fig. 3 Average foundation vertical settlement-time histories recorded in all tests

shown in Fig. 3. In this figure, positive displacements represent the settlement of foundation vertically downwards. During the injection of air, some settlements of shallow foundation happened due to the decrease of the effective stresses in the foundation soil. The mechanisms of settlement during this process were explained by Zeybek and Madabhushi (2016). The air-induced settlements observed in the partially saturated models are indicated by horizontal dashed lines. The results show that the vertical settlements of shallow foundations significantly decreased as the air bubbles were introduced into the initially liquefiable soil deposits. For instance, although the duration of the shakings was much longer in the partially saturated soil tests (PSL-1 and PSL-2), the foundation settled significantly less, compared to test with no remediation (FSL-1). After the same number of acceleration cycles (17), the total foundation settlements, including the air injection-induced settlements, were 948, 491 and $237 \mathrm{~mm}$ for FSL-1, PSL-1 and PSL-2, respectively. This indicates that the footing settlements were reduced by almost 48 and $75 \%$ following air injection during centrifuge tests PSL-1 and PSL-2. From these results, it appears that the reduction of foundation settlement did follow the reduction in the degree of saturation. Moreover, the introduction of air bubbles led the co-seismic foundation settlement rates to reduce dramatically.

The degree of saturation of soils and bearing pressure of the foundations were varied in different experiments. Therefore, the cumulative foundation settlement over 17 cycles against degree of saturation and bearing pressure histories are presented Fig. 4 to provide a direct comparison between the tests, and show the influence of a reduction in the degree of saturation on the trends of foundation settlement. In Fig. 4, it is possible to see that for a light foundation, the cumulative settlements reduce with a reduction in the degree of saturation, but below a degree of saturation of around $80 \%$ further reductions may be insignificant. In other words, a further reduction in the degree of saturation may not result in a marked decrease of foundation settlement. However, for a heavy foundation, it appears that the settlements continued to reduce up to a degree of saturation of $86 \%$. Further testing is required for the heavy foundation to establish the lower threshold of degree of saturation, below which partial saturation has no further benefit. 

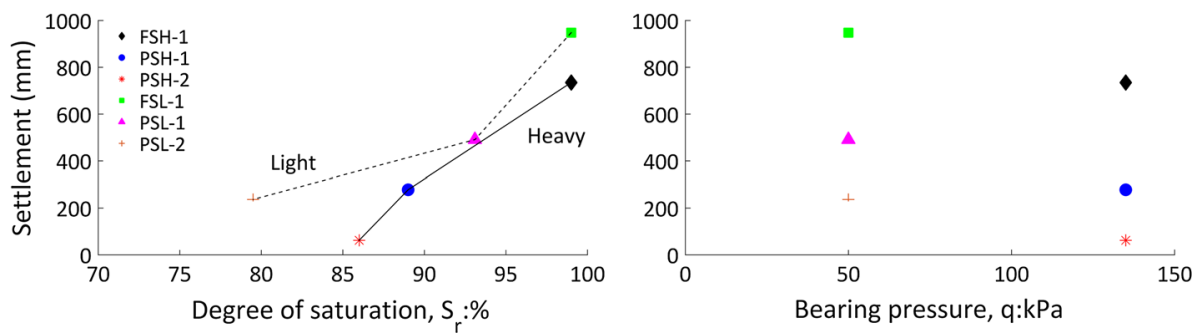

Fig. 4 Vertical settlement of foundation against degree of saturation and bearing pressure
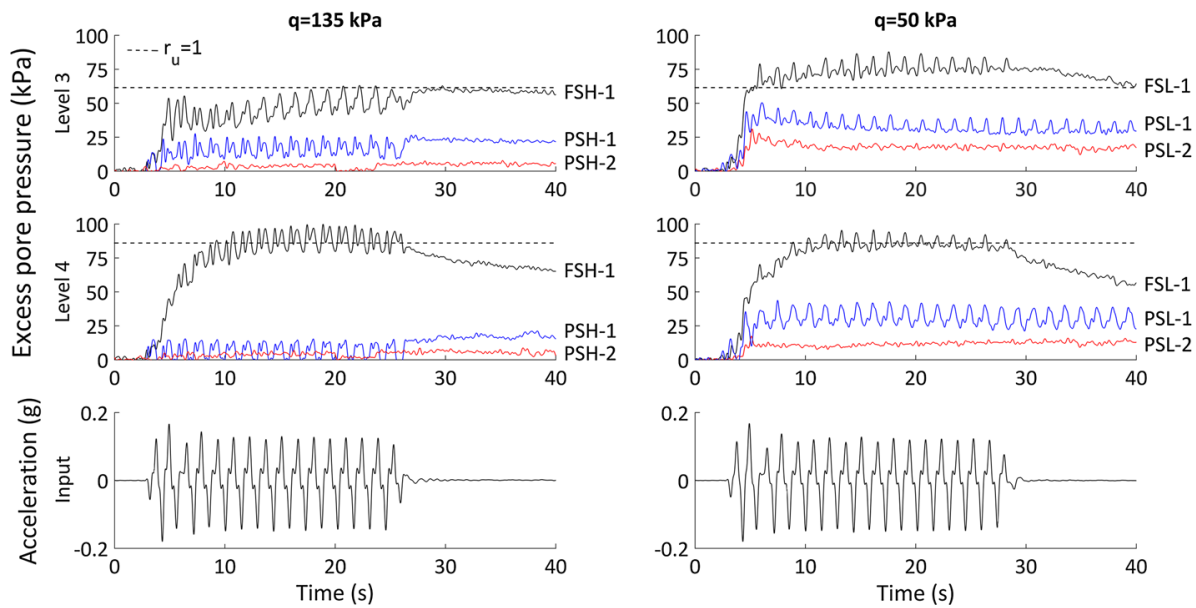

Fig. 5 Excess pore pressure-time histories measured beneath the shallow foundation (Sect. 2 in Fig. 1)

Comparing the results of nearly fully saturated tests (FSH-1 and FSL-1), increasing the bearing pressure of shallow foundations decreased the foundation settlement. The bearing pressure of heavy foundation increased the stress field underneath it and changed the excess pore pressure behaviour, as explained in the next section (see Fig. 5). The cyclic resistance of the foundation soil was probably increased due to the increased foundationinduced confining stresses. In parallel with this observation, it was also evident that the effect of degree of saturation was notably greater under the higher foundation-induced confining stresses, confirming the laboratory data from Okamura and Soga (2006). Therefore, it is suggested that the effect of degree of saturation on reducing foundation settlements is also dependent on the stress level, and the success of air introduction as a liquefaction mitigation is expected to be improved more beneath a heavier structure.

\subsubsection{Excess pore pressure}

The excess pore pressures measured at the middepth of soil layer beneath the shallow foundations and input acceleration data from tests FSH-1 and FSL-1 are shown in Fig. 5. Comparing the results, considerably smaller excess pore pressures were generated within the partially saturated soils than nearly fully saturated soils. The excess pore pressures in 
the nearly fully saturated soils rose to the initial effective stresses calculated without considering the foundation-induced stresses (maximum value of pore pressure ratio, $r_{u}=1$ in the free-field), but they did not reach in the partially saturated soils. Moreover, decreasing the degree of saturation of partially saturated soils slowed down the rate of excess pore pressure generation and reduced its magnitude further. The more the reduction in the degree of saturation led to the development of the lower excess pore pressures. The impact of this effect was however influenced by the confining pressure of foundations.

Comparing the excess pore pressures developed within the unimproved soils at Level 3, smaller excess pore pressures were generated under the heavy foundation (FSH-1) than light foundation (FSL-1). Moreover, at this level the generation of excess pore pressure to a maximum was more gradual and slower under the heavy foundation. Although the soil under higher confinement is capable of generating higher excess pore pressures for a given pore pressure ratio $r_{u}$, it requires larger cyclic shear strains to develop excess pore pressures. Under the selected input motions (with the identical properties), the resistance of soil to pore pressure generation is expected to be higher under the heavy foundation due to the larger foundation-induced confining stresses. Similar observations were made by Dashti et al. (2010). However, the magnitude and pattern of excess pore pressures were very similar at Level 4 in these tests, suggesting that the influence of foundation-induced confining stresses on the pore pressure generation became negligible at locations away from the foundation.

The comparison of the excess pore pressures developed within the partially saturated soils also shows that consistent with the settlement data, the influence of degree of saturation of soil on its resistance to pore pressure generation depends on the stress level. The resistance to pore pressure generation increased as the degree of saturation was reduced, and this was more pronounced under the higher confining pressure of foundations and deeper layers of soils.

\subsubsection{Acceleration amplification/attenuation}

The degree of saturation influenced the settlement and pore pressure behaviour beneath shallow foundations, which in turn affected the acceleration-time histories experienced within the soil column and foundations. These accelerations were explicitly investigated, and amplification/attenuation ratios were calculated on a cycle by-cycle basis. These ratios were found by dividing the maximum positive and negative acceleration of a given accelerometer in each cycle by the maximum positive and negative acceleration of the corresponding input accelerometer in the corresponding cycle.

The amplification/attenuation ratio-cycle number history recorded during the tests beneath the light foundation (FSL-1, PSL-1 and PSL-2) is presented in Fig. 6 (the models had a similar maximum input acceleration, but different initial degrees of saturation). The acceleration records during the test FSL-1 show that as the soil liquefied the amplitude of foundation acceleration and soil acceleration in the both positive and negative directions was significantly reduced. The attenuation ratio nearer the surface (Level 2) for instance was 0.18 of the input in the positive direction around cycle 3 . At the deeper layer (Level 4), an attenuation occurred until the first 5 cycle, but then there was some amplification, suggesting the dilation of soil and re-gaining some strength. On the other hand, in the tests PSL-1 and PSL-2, the soil layers at Level 2 and Level 4 did not liquefy, and in turn a notable attenuation of foundation and soil acceleration did not occur. In test PSL-1, slight attenuation and amplification of foundation acceleration were apparent in the positive and negative direction, respectively. Moreover, the amplification/attenuation ratio was not 

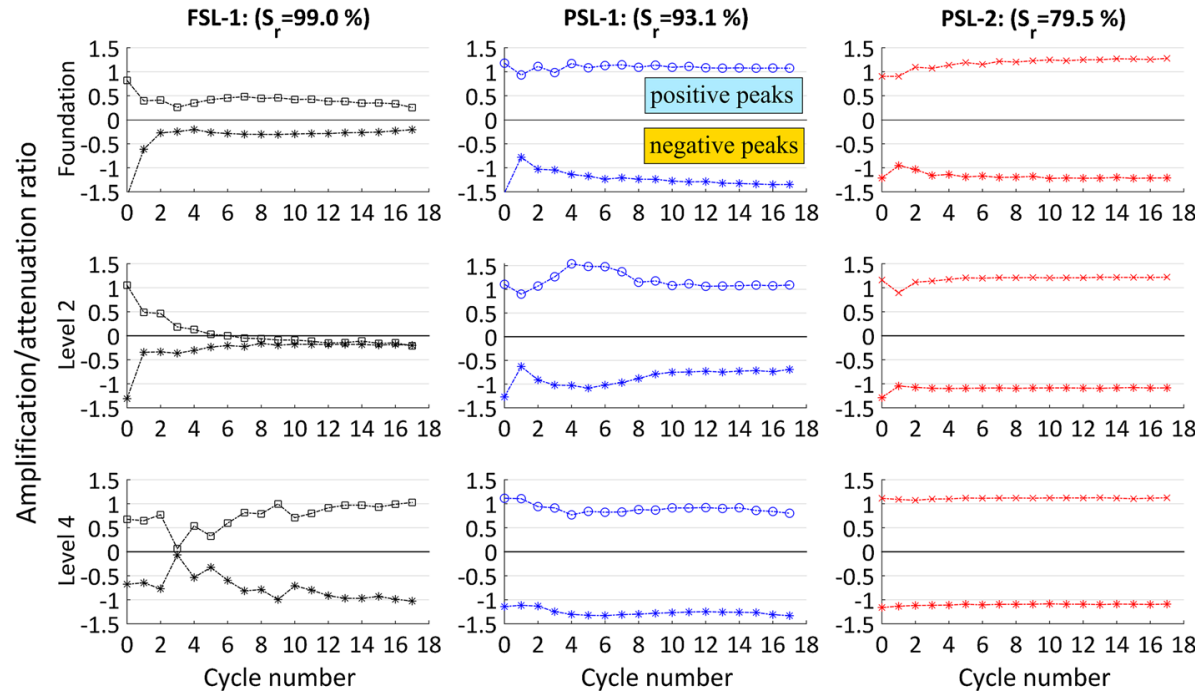

Fig. 6 Amplification/attenuation of light foundation and foundation soil (Sect. 2 in Fig. 1) in tests with different degrees of saturation
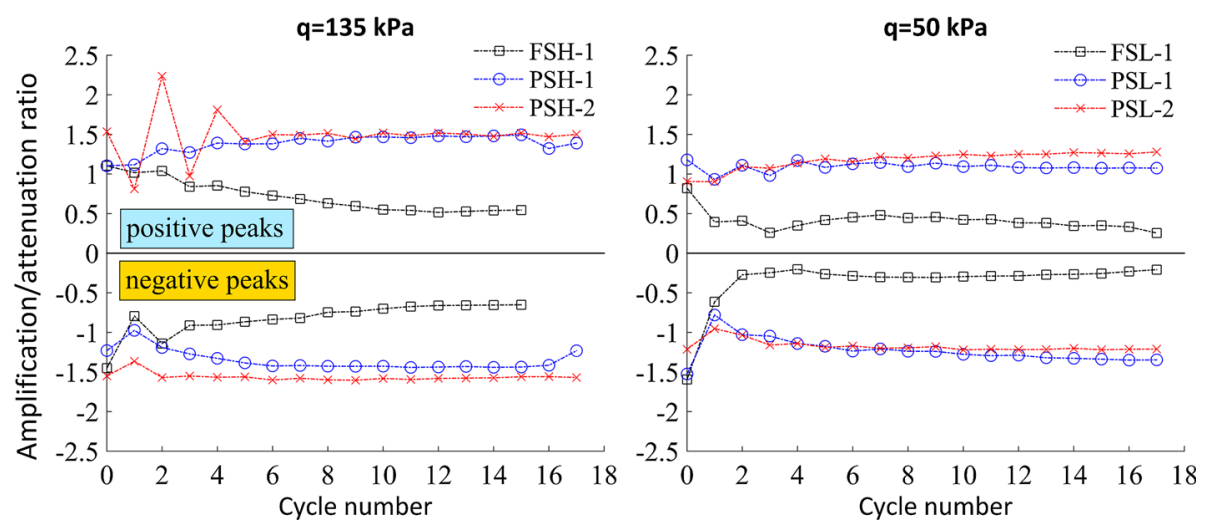

Fig. 7 Amplification/attenuation ratios for heavy and light foundation accelerations for all tests

constant over the duration of earthquake at some soil layers. However, further decrease in the degree of saturation of soils (PSL-2) entirely prevented the attenuation of foundation and soil acceleration, and oppositely it resulted in some amplification.

Figure 7 compares the amplification/attenuation ratios of heavy and light shallow foundations. It is observable that heavy foundation suffered less attenuation than light foundation in the nearly fully saturated soil tests. Similarly, larger accelerations were passed to the heavy foundation than light foundation in the air-injected partially saturated soil tests. This observation therefore indicates that the influence of degree of saturation on the magnitude of accelerations transmitted to the foundations is dependent on the bearing pressure of foundations. The increased stress level beneath the much heavier foundation together with the decrease in the degree of saturation of soil probably allows for the 
formation of an unliquefied and much stiffer soil zone. Therefore, much larger accelerations from this bearing stratum can be transmitted to the foundation. Balakrishnan and Kutter (1999) explained how the natural period of the soil deposits changes when the stiffness of soils alters. They claimed that increasing the stiffness of soil deposit may increase the ground motion amplification, depending on the predominant period of ground motions.

\subsection{Response in the free-field}

\subsubsection{Settlement}

The free-field responses were measured at locations sufficiently distant from the foundations. Therefore, soil structural interaction effects were considered to be negligible. The long-term histories of settlement measured beneath the heavy foundation (Sect. 2 in Fig. 1) and in the free-field (Sect. 3 in Fig. 1) were shown in Fig. 8. The contribution of airinduced, co-seismic and post-seismic settlements (separated by two vertical dashed lines) to the overall settlements were explicitly presented in this figure. It is obvious that in the nearly fully saturated and partially saturated soils the settlement of foundation occurred both during and after the seismic event, but post-seismic settlements were significantly smaller than the co-seismic settlements. The post-seismic foundation settlements considerably reduced with decreasing degrees of saturation. In the free-field, significant volume change happened during the cyclic loading, and this was more pronounced in the case of partially saturated soils. A decreasing trend in the post-seismic free-field settlements (due to re-consolidation associated with the dissipation of excess pore pressures) was observed with decreasing degrees of saturation for the evaluated tests.

In the case of large differential settlement between the soil surface and foundation, the structures can be unusable although they are undamaged by rotation or earthquake shaking. The comparison of the foundation and free-surface settlements in Fig. 8 reveals that the
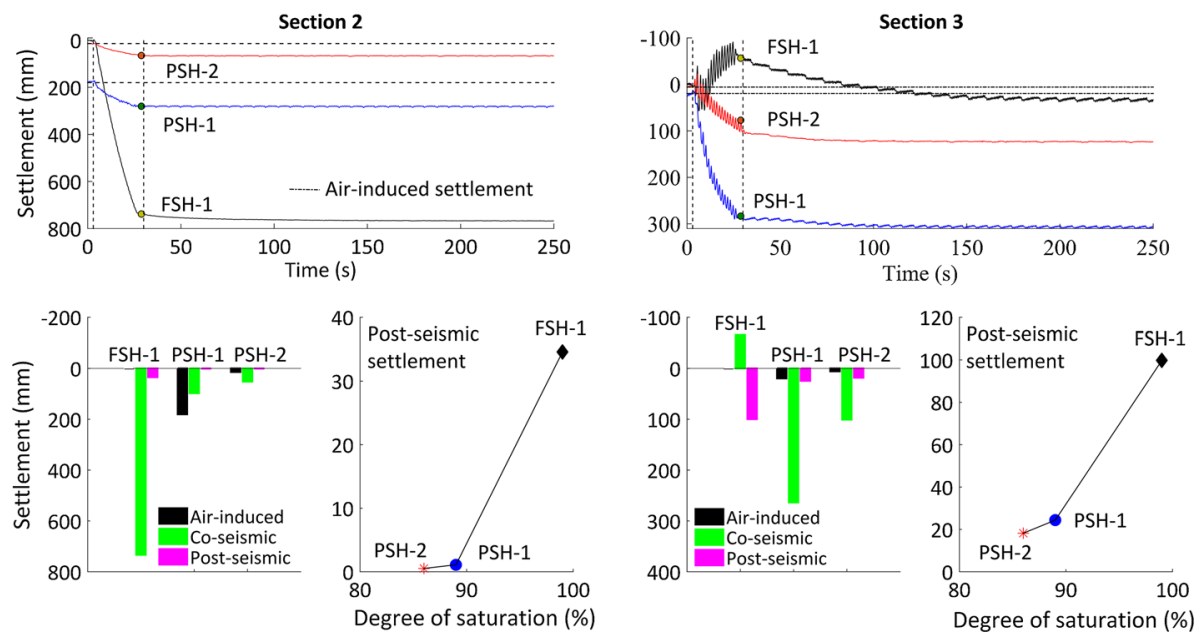

Fig. 8 Long-term foundation (Sect. 2) and free-field (Sect. 3) settlements during the tests with heavy foundation 
differential settlements were reduced significantly by air injection, and further reduction in the degree of saturation resulted in even less differential settlement.

\subsubsection{Drainage}

The hydraulic gradients recorded in the free-field (Sect. 3 in Fig. 1) for all tests are presented in Fig. 9 to investigate the effect of degree of saturation on the dissipation behaviour of soils. In this figure, hydraulic gradients were calculated using the difference in excess pressure head between two relevant points and the distance between them. A positive hydraulic gradient denotes upwards flow. The co-seismic behaviour (zone A) is separated from the post-seismic dissipation behaviour (zone B) by two vertical dashed lines.

The results of the tests with $S_{r}=99.0 \%$ demonstrated that very high excess pore pressure gradients were generated during shaking. The dissipation of excess pore pressures started from the base where the excess pore pressures had nowhere to dissipate and it was only permitted from the surface. Therefore, the flow of pore fluid was always upwards in all locations after the shaking ceased. The pore-fluid flow due to the excess pore pressure gradients usually began after the shaking ceased, except for soils at Level 2-3 and Level 3-4 under light foundation where it started right before the end of shaking. The pore fluid flow continued until pore pressures reached the equilibrium throughout the soil. The soils at the low effective stresses (particularly Level 1 and Level 2) remained in a weakened state for longer.

Comparing with the nearly fully saturated soils, much smaller excess pore pressure gradients were generated during shaking in the air-injected partially saturated soils. Some downwards flows were apparent in some layers, particularly between Level 3 and 4 . The hydraulic gradients were generally small when the earthquakes finished, and they dropped to zero much more quickly than the nearly fully saturated soils, when considering the tests with a heavy foundation. Moreover, although the duration of earthquakes in the air-injected partially saturated soils beneath the light structure was much longer, the hydraulic
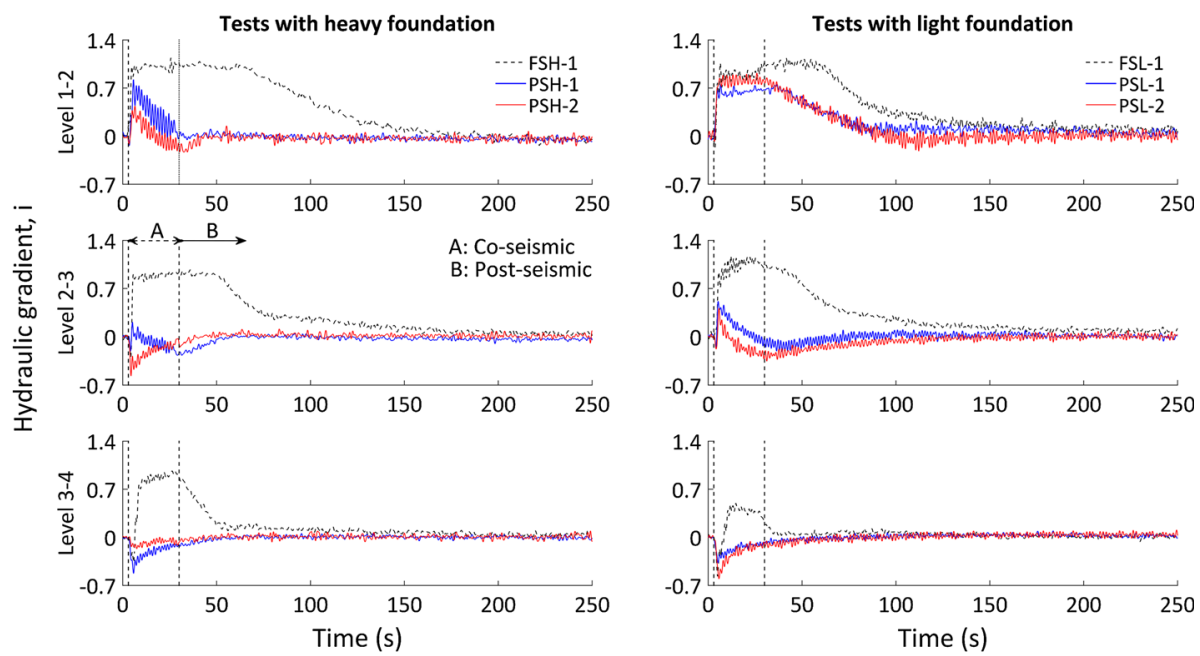

Fig. 9 Hydraulic gradients measured in the free-field (Sect. 3 in Fig. 1) during and after earthquakes 
gradients in these tests also dropped to zero quicker or at the same time as the nearly fully saturated case. Overall, the pore fluid flow due to the excess pore pressure gradients ceased, and equilibrium of the pore pressures throughout the soil was maintained much earlier in the partially saturated soils.

Figure 10 presents the 2D digital images taken on the side (front window) of the nearly fully saturated soil (FSH-1) and partially saturated soil (PSH-2). The images were processed with the image processing toolbox-MATLAB and shown in Fig. 10a, b to illustrate how the colour of soils tended to change with the injection of air. It is observed that the colour of soil was much lighter in the portion of soils with air bubbles. The shape of effective partially saturated zone, indicated by the broken curves, was an almost parabolic $\mathrm{U}$, and it was approximately symmetrical. It however appears that the air bubbles were retained erratically, and the partial saturation was not completely uniform, even within the effective partially saturated zone. The enlarged images of nearly fully and partially saturated soils presented in Fig. 10c, d further confirm the colour difference between them and the presence of air bubbles. It also shows that the retained air bubbles were in the occluded form within the pore fluid and their sizes were very small (smaller or same order of grain size).

It is obvious from the aforementioned results that the response of air-injected partially saturated soils differed from the response of nearly fully saturated soils. The free-field seismic settlements in nearly fully saturated soils predominantly occurred during the shaking, and these co-seismic settlements can be attributed to the partial drainage (Dashti et al. 2010). Similarly, the majority of the free-field settlements were co-seismic in the airinjected partially saturated soils, but these settlements can be attributed to the increased compressibility of the pore fluid within the soil specimens (Okamura and Soga 2006). It is also observable that the mechanisms of excess pore pressure generation and dissipation in the nearly fully saturated and air-injected partially saturated soils were quite different, but the latter is particularly complicated. It is known that excess pore pressures can generate only where the voids are filled with pore fluid. The presence of air bubbles in the partially saturated soils can either prevent or reduce the excess pore pressure generation, depending on the degree of saturation and initial pressure of air bubbles. The observed trends show
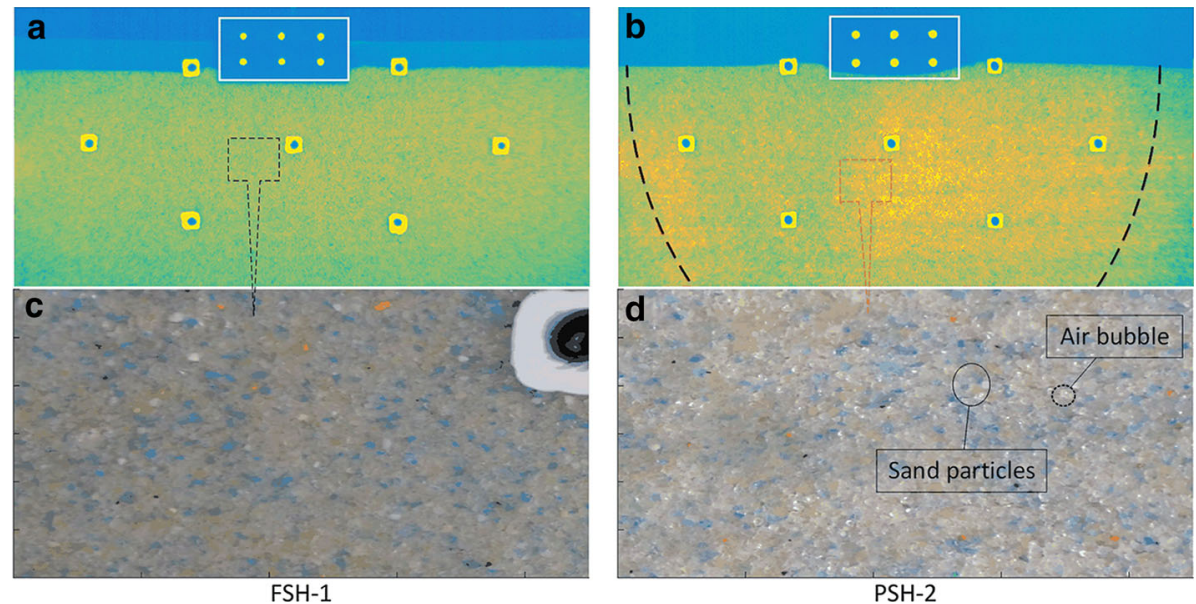

Fig. 10 The colour of nearly fully saturated soil (FSH-1) and partially saturated soil (PSH-2)-Brighter yellows indicate lower degrees of saturation 
that much smaller excess pore pressures were generated in the partially saturated soils, with air bubbles absorbing the generated excess pore pressures by reducing their volumes. However, these bubbles probably affected the dissipation of excess pore pressures since they could decrease the hydraulic conductivity of the soils. Moreover, it is assumed that the complexity of the dissipation behaviour in these soils was increased further by the nonuniform distribution of air bubbles within the soil specimens, and further research will be needed to explain the cause.

\subsection{Deformation mechanisms}

The influences of degree of saturation on the settlement, excess pore pressure and acceleration responses were quantitatively assessed thus far. Examining the liquefaction-induced deformation mechanisms can provide better insight into the relative importance of degree of saturation for the overall performance of the structures with the shallow foundations. Figure 11 presents the images taken after 17 cycles of acceleration during some tests. In the nearly fully saturated soils (FSH-1 and FSL-1), a very deep layer of deformation took place in the liquefiable sand layer, with foundations settling more than the adjacent ground and the ground adjacent to the foundations settling more than the free field. The width of the surface settlement trough (profile) was much narrower beneath the light foundation. Very large lateral movements of the coloured sand columns which are indicative of deviatoric deformations and evenly distributed throughout the liquefiable layer were apparent (FSL-1). These lateral deformations increased from bottom to the surface of soil. On the other hand, in the partially saturated soils (PSH-2 and PSL-1), only shallow layer of deformation took place in the soil layer. The horizontal soil movement was concentrated only at the shallow soil layer, and its magnitude was very small.

The deformation mechanisms developed during the experiments with the heavy foundation were demonstrated by Zeybek and Madabhushi (2016), with a particular focus on the alteration of deformation mechanisms with the injection of air. It was found that deviatoric soil deformations and lateral movement of soils were significantly reduced with
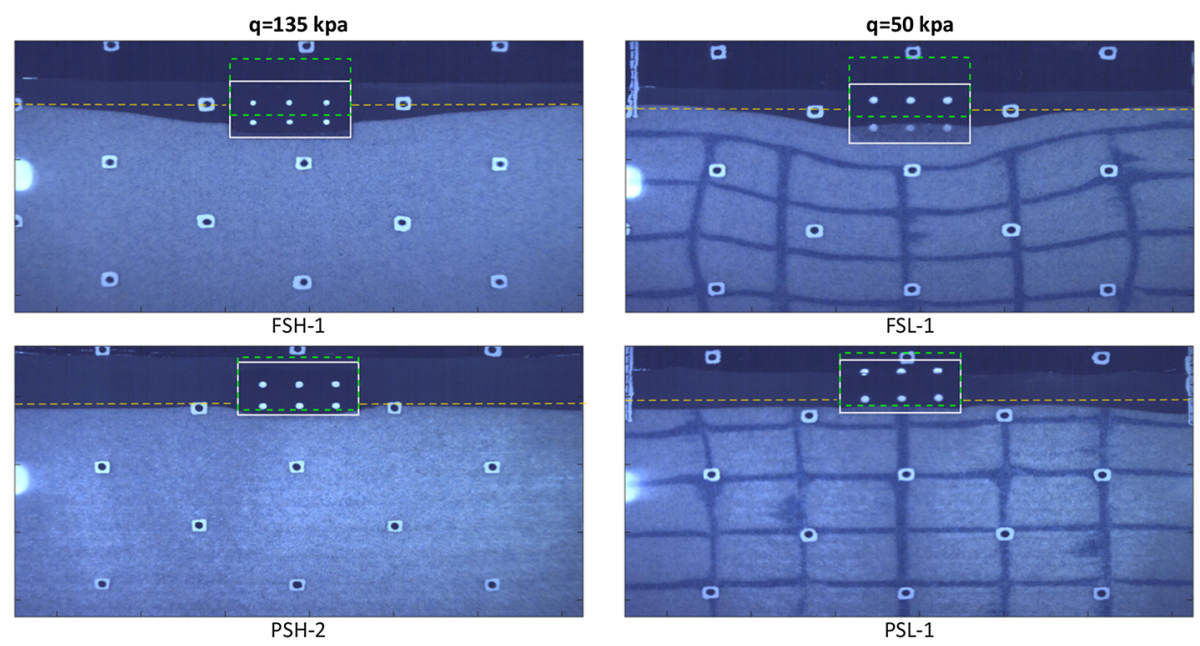

Fig. 11 The shape of deformation after 17 cycles of acceleration (the dashed lines represent the initial locations) 
this particular liquefaction remediation scheme. In this paper, the deformation mechanisms developed beneath the light foundation were shown, with an interest in the impact of the degree of saturation on the volumetric and deviatoric strains. The accumulated displacements occurring through 17 acceleration cycles beneath the light foundation are shown in Fig. 12. The displacements illustrated at the same scale in Fig. 12a-c visually illustrate that the deformations in the partially saturated soils (PSL-1 and PSL-2) were significantly less than those in the nearly fully saturated soil (FSL-1). It is obvious that the deviatoric and volumetric soil strains and the consequent soil displacements were both apparent in test FSL-1, but the deviatoric strains were more dominant to the settlement of the shallow foundation. This is evident from the strong tendency of horizontal movements in soil underneath the foundation and formation of the deformation wedges. The depth of liquefaction increased to a level where a bearing capacity failure mechanism formed. On the other hand, the magnified displacement vectors in Fig. 12e, f reveal that the soil movements were considerably smaller, and the mobilisation of a bearing capacity failure mechanism was not the case in tests PSL-1 and PSL-2. The positive volumetric strains (associated with the increased compressibility of the air-injected soil) increased, leading to vertical soil deformations, and the majority of the settlements in these soils happened due to these volumetric strains. However, even with this increase the overall deformations were still significantly smaller than those observed in the nearly fully saturated soil. The depth of

a

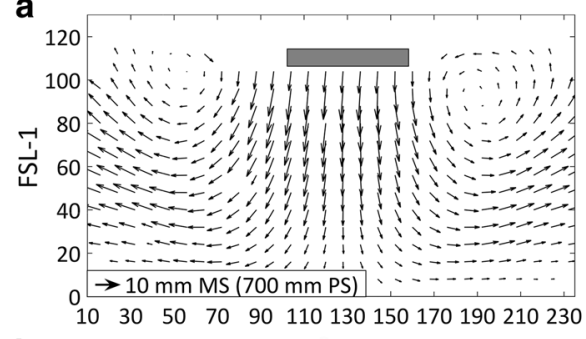

b

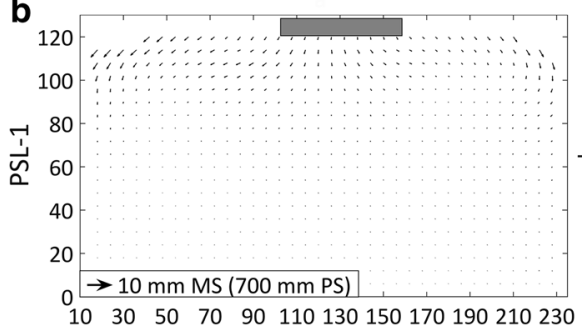

c

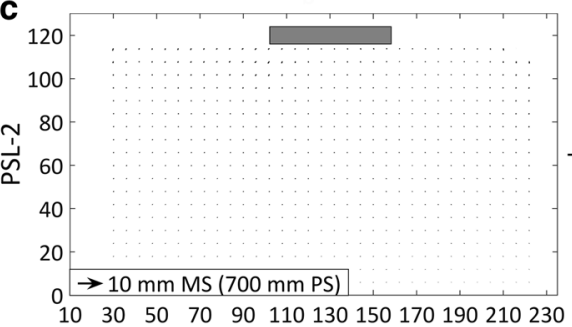

d
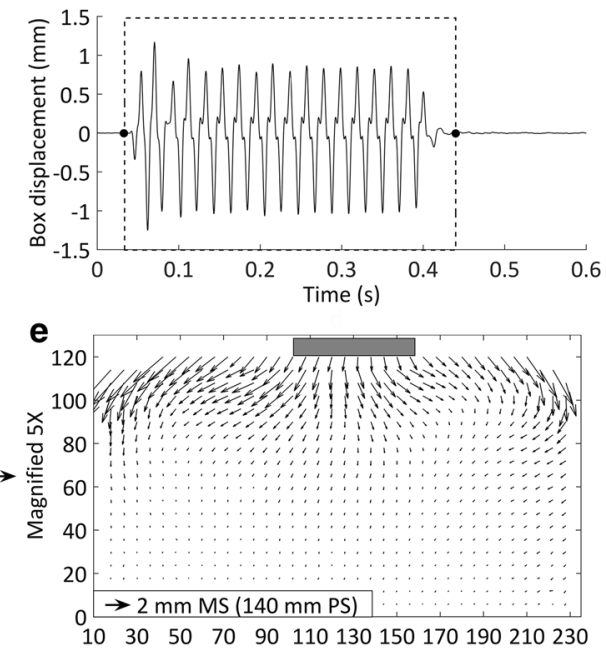

f

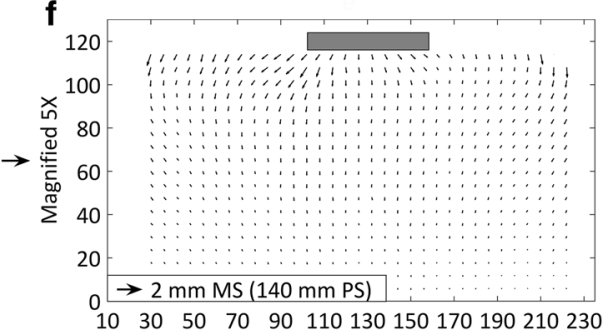

Fig. 12 Accumulated soil deformations over 17 acceleration cycles for a light foundation with $q=50 \mathrm{kPa}$ 
liquefaction was limited by the air injection, and this effect increased as the degrees of saturation of soil reduced.

The degree of saturation of partially saturated soils has a significant impact on the shear strains and consequent horizontal soil movements developed during the shakings. As shown in Fig. 13, the accumulated shear strains occurring over 17 acceleration cycles decreased as the degree of saturation of soils reduced. A decreasing trend in the shear strains with decreasing degrees of saturation can explain the reason for the further reduction in the horizontal movement of foundation soil and shear-type movements in the partially saturated soil with smaller degree of saturation (PSL-2).

The effect of degree of saturation on the soil deformations is investigated in the case of different bearing pressures. The displacement vectors of Fig. 14 show the deformations corresponding to the first 17 cycles of earthquake loading beneath the shallow foundations with two different bearing pressures. Since the deformations in partially saturated soil tests (PSH-2 and PSL-2) were relatively very small, the magnitude of displacements is magnified by 20 times. Comparing with the nearly fully saturated soils (FSH-1 and FSL-1), the magnitude of soil displacements was significantly smaller, and the majority of soil deformations occurred vertically downwards, with little lateral and outwards soil movements in the partially saturated soils (PSH-2 and PSL-2). The lateral movements of soil into the free-field were significantly reduced in these soils, and they were concentrated only at the shallower soil layers. These findings were well-consistent with the presented data above. The comparison between two partially saturated tests showed that the magnitude of outwards soil movements from the foundation at the very surface was relatively smaller in test PSH-2 although the degree of saturation of soil in this test was much higher. This behaviour can be attributed to the increased stress regime in the ground due to the larger foundation-induced confinement. It is suggested that the foundation-induced stresses
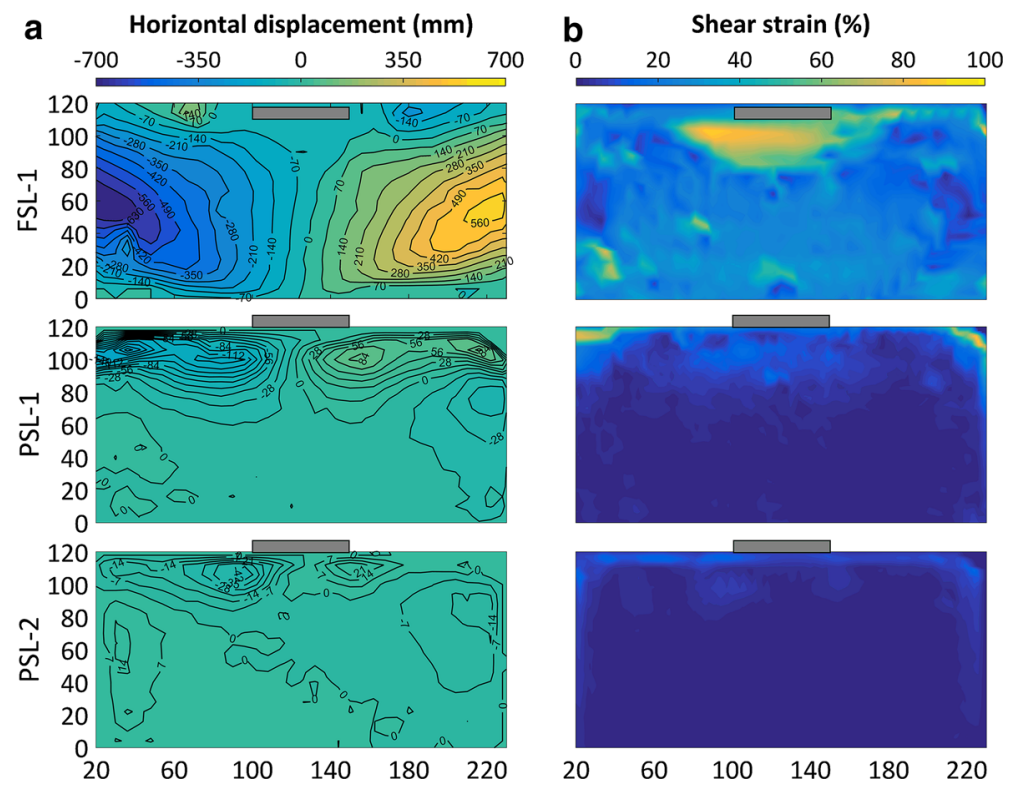

Fig. 13 Accumulated horizontal displacements and shear strains over 17 acceleration cycles for a light foundation with $\mathrm{q}=50 \mathrm{kPa}$ 

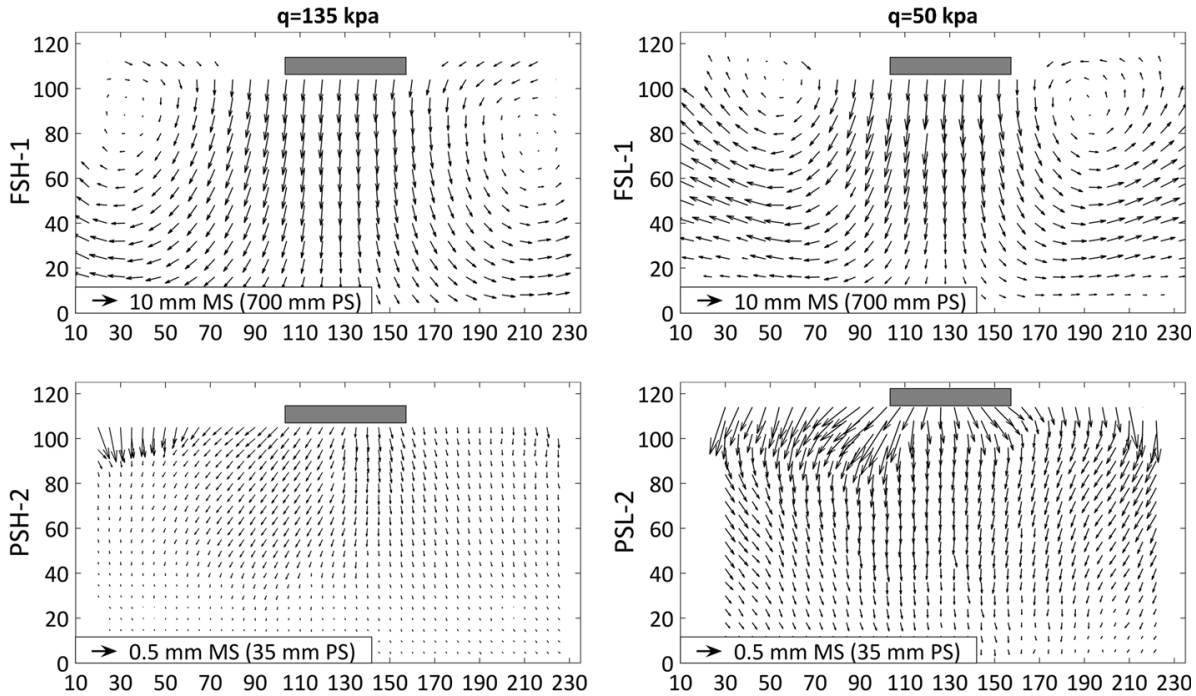

Fig. 14 Cumulative soil deformations over 17 acceleration cycles for foundations with different bearing pressures

should be incorporated in investigating the influence of degree of saturation on the potential soil and foundation movements.

The comparison of the soil movements in the nearly fully saturated tests (FSH-1 and FSL-1) provides an insight into the effect of foundation-induced stresses indeed. In both tests, significant deformations and a typical bearing capacity failure mechanism were evident beneath and under both edges of the foundation. However, lateral soil movements were much larger beneath the light foundation than the heavy foundation. This observation may indicate that the development of horizontal soil movements is directly associated with the stress level and soil stiffness beneath the foundation. Foundation-induced confinement due to larger bearing pressure reduces the horizontal soil movement. This eventually provides a reduction in the liquefaction-induced settlement of shallow foundations.

\section{Conclusions}

The effect of degree of saturation on the cyclic liquefaction behaviour of air-induced partially saturated soils beneath shallow foundations was investigated through a series of geotechnical centrifuge tests. These centrifuge tests provided insight into the quantitative influence of degree of saturation on the settlement, pore pressure and acceleration responses of partially saturated soils and shallow foundations founded on these soils.

It was found that generation and dissipation of excess pore pressures and consequent liquefaction-induced settlements of shallow foundations were a strong function of the degree of saturation. The observed excess pore pressures and settlements showed a decreasing trend with a reduction in the degree of saturation at a range between 80 and $99 \%$. Decreasing the degree of saturation of soils proved to be significantly beneficial to the overall shallow foundation response since the seismic foundation settlement drastically reduced. It minimized the development of high excess pore pressures and liquefaction 
susceptibility of soil, particularly at the higher confining stresses. Moreover, as the degree of saturation reduced, post-seismic free-field settlements (volumetric strains) due to reconsolidation dramatically decreased. Similarly, the reduction in the degree of saturation had a decreasing impact on the post-seismic foundation settlement, enhancing the postshaking bearing capacity. Reducing the degree of saturation was, though, found to intensify the accelerations transmitted to the foundations through unliquefied soil zones.

The displacement vectors fields and shear strain distributions were used to elucidate the effect of degree of saturation on the deformation mechanisms. The results emphasised that the shear strains and consequent seismic horizontal-vertical soil movements significantly reduced with a decreasing degree of saturation. The reduction in the degree of saturation of soils limited the depth of liquefied soil layer and increased the resistance of soil to the bearing capacity failure.

The influence of degree of saturation on the soil and foundation response was found to be dependent on the stress level. Under the higher confining pressure of foundations and deeper layer of soils, the decrease in the degree of saturation of soils was potentially more influential on reducing the foundation settlements, increasing the resistance to pore pressure generation and amplifying the foundation accelerations. Therefore, this parameter should be incorporated while studying the impact of degree of saturation on the basic aspects of foundation performance and corresponding soil deformation mechanisms.

Acknowledgments The first author wishes to acknowledge the financial contribution of the Ministry of National Education (M.E.B.) of Turkey during the course of this study. The writers would also like to thank those, particularly the technicians, at the Schofield Centre of Cambridge University for their assistance during the centrifuge tests.

Open Access This article is distributed under the terms of the Creative Commons Attribution 4.0 International License (http://creativecommons.org/licenses/by/4.0/), which permits unrestricted use, distribution, and reproduction in any medium, provided you give appropriate credit to the original author(s) and the source, provide a link to the Creative Commons license, and indicate if changes were made.

\section{References}

Balakrishnan A, Kutter BL (1999) Settlement, sliding, and liquefaction remediation of layered soil. J Geotech Geoenviron Eng ASCE 125(11):968-978

Dashti S, Bray JD, Pestana JM, Riemer M, Wilson D (2010) Mechanisms of seismically induced settlement of buildings with shallow foundations on liquefiable soil. J Geotech Geoenviron Eng ASCE 136(1):151-164

Kutter BL (2013) Effects of capillary number, bond number, and gas solubility on water saturation of sand specimens. Can Geotech J 50(2):133-144

Madabhushi SPG (2014) Centrifuge modelling for civil engineers. Taylor \& Francis, London

Madabhushi SPG, Schofield AN, Lesley S (1998) A new stored angular momentum (SAM) based earthquake actuator. In: Proceedings of Centrifuge 98, Tokyo, Japan: Balkema, pp 111-116

Marasini N, Okamura M (2015) Air injection to mitigate liquefaction under light structures. Int J Phys Model Geotech 15(3):129-140

Mitchell JK, Santamarina JC (2005) Biological considerations in geotechnical engineering. J Geotech Geoenviron Eng 131(10):1222-1233

Okamura M, Soga Y (2006) Effects of pore fluid compressibility on liquefaction resistance of partially saturated sand. Soils Found 46(5):695-700

Okamura M, Takebayashi M, Nishida K, Fujii N, Jinguji M, Imasato T, Yasuhara H, Nakagawa E (2011) Insitu desaturation test by air injection and its evaluation through field monitoring and multiphase flow simulation. J Geotech Geoenviron Eng ASCE 137(7):643-652 
Schofield AN (1981) Dynamic and earthquake geotechnical centrifuge modelling. In: Proceedings of International Conference on Recent Advances in Geotechnical Earthquake Engineering and Soil Dynamics, University of Missouri-Rolla, MO, USA, pp 1081-1100

Stringer ME, Madabhushi SPG (2009) Novel computer-controlled saturation of dynamic centrifuge models using high viscosity fluids. Geotech Test J 32(6):559-564

Takemura J, Igarashi R, Izawa J, Okamura M (2008) Centrifuge model tests on soil desaturation as a liquefaction countermeasure. In: Proceedings of the 5th International Conference on Urban Earthquake Engineering, Tokyo Institute of Technology, Tokyo, Japan, pp 269-274

Tsukamoto Y, Ishihara K, Nakazawa H, Kamada K, Huang YN (2002) Resistance of partly saturated sand to liquefaction with reference to longitudinal and shear wave velocities. Soils Found 42(6):93-104

White DJ, Take WA, Bolton MD (2003) Soil deformation measurement using particle image velocimetry (PIV) and photogrammetry. Géotechnique 53(7):619-631

Yegian MK, Eseller-Bayat E, Alshawabkeh A, Ali S (2007) Induced-partial saturation (IPS) for liquefaction mitigation: experimental investigation. J Geotech Geoenviron Eng ASCE 133(4):372-380

Yoshimi Y, Yanaka K, Tokimatsu K (1989) Liquefaction resistance of a partially saturated sand. Soils Found 29(3):157-162

Zeybek A, Madabhushi SPG (2016) Influence of air injection on the liquefaction induced deformation mechanisms beneath shallow foundations. Soil Dyn Earthq Eng (under review) 